\title{
HG-LG Mode Conversion with Stressed 3-Mode Fibers under Polarization
}

\author{
Henning Soller \\ Institut für Theoretische Physik, Rupprecht-Karls-Universität Heidelberg, Heidelberg, Germany \\ Email: hsoller@googlemail.com
}

Received September 11, 2012; revised October 14, 2012; accepted October 25, 2012

\begin{abstract}
We have coupled an upright HG mode into a fiber-optic waveguide and used the application of stress to generate a Laguerre-Gaussian laser mode. We have generalized previous results by McGloin et al. by using a polarized input beam, a true 3-mode fiber and by applying the stress on a stripped piece of the optical waveguide. These generalizations are necessary in order to perform quantum information experiments and obtain reliable information on the stress imposed on the optical fiber.
\end{abstract}

Keywords: Mode Conversion; Stress; Optical Fiber; Laguerre-Gaussian Laser Mode

\section{Introduction}

Recently the production of Laguerre-Gaussian (LG) laser modes has again attracted considerable interest [1]. It arose mainly from the fact that these modes have a well defined orbital angular momentum equal to $l \hbar /$ photon [2] which may be used to transfer information. The angular momentum content of LG beams has been transferred successfully to microscopic particles [3] and a comparison of the spin angular momentum has shown it to be $l \hbar$ / photon $([4,5])$. Orbital angular momentum is of special interest for quantum information processing since different Fourier components of LG beams have been shown to be independently addressable [6]. Also their importance for producing heralded single photons in arbitrary spatial modes has been addressed [7]. Four different possibilities for creating LG modes have been discussed. First, LG beams can be produced from a laser directly [8]. Furthermore, there are two classes of mode converters for Hermite-Gaussian (HG) laser modes that can be obtained more easily in open cavity lasers. The first type of mode converter includes spiral phase plates $[9,10]$ and computer-generated holographic converters $[11,12]$. In these devices, an azimuthal phase dependence is introduced into an $\mathrm{HG}_{00}$ beam. The third class of converters is based on cylindrical lenses [13,14]. Unlike the methods using spiral phase plates or holographic converters this method allows to produce pure LG modes. Finally, the fourth type of converter uses a stressed fiber-optic waveguide and an HG mode input laser [1]. Compression of the fiber optic waveguide causes the non-cylindrically symmetric $\mathrm{HG}_{10}$ and $\mathrm{HG}_{01}$ modes to experience differing phase velocities as they propagate through the fiber. The nonzero relative phase shift is controllable by the magnitude and direction of the applied stress. If these phase shifts are such that the two HG modes undergo phase shifts that differ by $90^{\circ}$ the resultant field distribution is an LG mode with $l=1$ and $p=0$.

In this paper we want to show how the result of [1] can be generalized in three ways: first, in the previous experiment the stress has been put onto the fiber directly. We wanted to see whether it is possible to strip the fiber and consequently allow to apply stress in a more controlled manner. Second we did not want to use an HG mode that is coupled in at a specific angle but just to use an upright HG mode and see whether leaking of one HG mode into the other inside the optical fiber can produce the required angle by itself. Finally quantum information experiments (e.g. [7]) require to do the experiments also for polarized input beams and fibers as close to a 3-mode fiber as possible. We will illustrate the possibility to produce Laguerre-Gaussian laser modes with heralding photons in the second part of this report.

\section{Experiment}

In this research we used a fiber of 1-m length. Using different types of optical fibers we made sure to have a real 3-mode fiber. Figure 1 shows the experimental apparatus used to convert the HG input beam. The input laser was forced to oscillate in an $\mathrm{HG}_{10}$ mode by the inclusion of an intracavity cross wire. The output beam is polarized and expanded to fill the back aperture of a microscope 


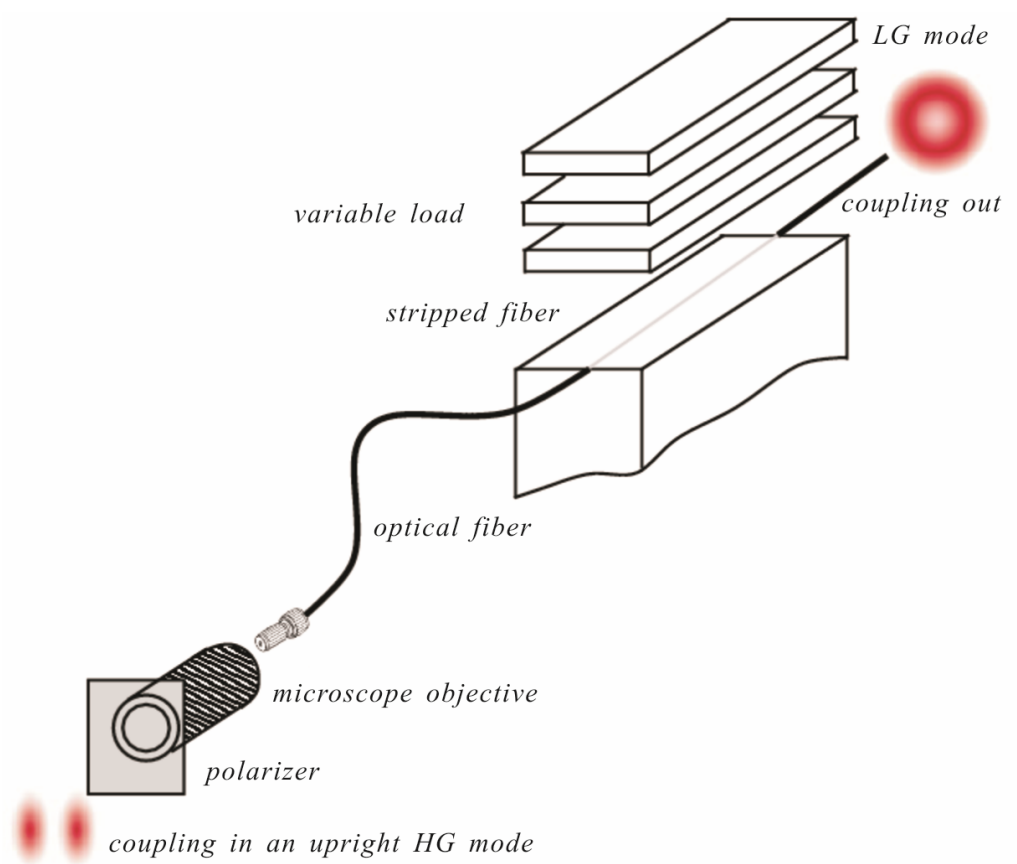

Figure 1. The figure shows the experimental arrangement. We couple in an upright HG mode, that leaks into the rotated mode and can therefore be transformed into an LG mode. The incoming mode is polarized and coupled into an optical waveguide via a microscope objective. The stress is applied via lead blocks on the stripped fiber.

objective mounted on an $\mathrm{x}, \mathrm{y}, \mathrm{z}$ stage to couple the beam into a 3-mode optical fiber $(780 \mathrm{~nm})$. The fiber was stressed over a $100 \mathrm{~mm}$ region by lead weights put directly on top of the stripped fiber. One lead block on a stage was put below the fiber and two old fiber pieces of the same kind were used on the sides to get homogeneous stress.

The input mode of the optical fiber before the compression stage may be assumed to be a 45 degree rotated HG mode

$$
\left|\mathrm{HG}_{45^{\circ}}\right\rangle=\left|\mathrm{HG}_{01}\right\rangle+\left|\mathrm{HG}_{10}\right\rangle,
$$

where we leave out normalization factors. Depending on the stress induced a relative phase shift between the modes occurs that may lead to a relative phase factor of $\mathrm{e}^{i \pi / 2}=i$ so that

$$
\left|\mathrm{HG}_{45^{\circ}}\right\rangle \rightarrow\left|\mathrm{HG}_{01}\right\rangle+i\left|\mathrm{HG}_{10}\right\rangle=\left|\mathrm{LG}_{0}^{1}\right\rangle
$$

Further stress leads to relative phase shift of $\mathrm{e}^{i 3 \pi / 2}=-i$ so that [1]

$$
\left|\mathrm{HG}_{45^{\circ}}\right\rangle \rightarrow\left|\mathrm{HG}_{01}\right\rangle-i\left|\mathrm{HG}_{10}\right\rangle=\left|\mathrm{LG}_{0}^{-1}\right\rangle,
$$

Consequently both linear momentum components of the first LG mode can be addressed using the compression method.

The overall efficiency of this mode converter has been measured to be approximately $2 \%-3 \%$. We should stress that the usage of a 3-mode fiber instead of the 1.06 $\mu \mathrm{m}$ fiber used in [1] considerably complicates the cou- pling but only this way higher order LG modes can be discarded. We used different methods for stripping the optical waveguide, e.g. burning the cladding, chemical etching and mechanical stripping. Both chemical etching and burning have caused considerable damage to the optical waveguide and only mechanical stripping has proven to allow for controllable operation of the fiber afterwards.

We needed about $1.5 \mathrm{~kg}$ of lead to observe the mode change to $\mathrm{LG}_{0}^{1}$, again also under polarization. After the addition of $1.5 \mathrm{~kg}$ of further lead blocks the output went back to an HG mode rotated approximately $90^{\circ}$ with respect to the input mode. No further mode changes have been observed even under extreme stress of about $11 \mathrm{~kg}$ of lead. However, it is remarkable that the fiber works even under such extreme conditions.

\section{Heralded Single Photons}

This setup may now be used in combination with the Sagnac interferometer described in [7] to produce heralded single photons, see Figure 2. In the setup a $45^{\circ}$ rotated HG laser pump is used.

We had shown above that one could also use a different rotation and an optical fiber to achieve the required rotated beam. Using the nonlinear crystal one achieves that the resulting input state is predominantly a superposition of the vacuum and a two-photon state $\left|\Psi_{2 p h}\right\rangle$ that we can use to achieve the heralding of the photons. Since the vacuum state is not relevant for us we focus on the 


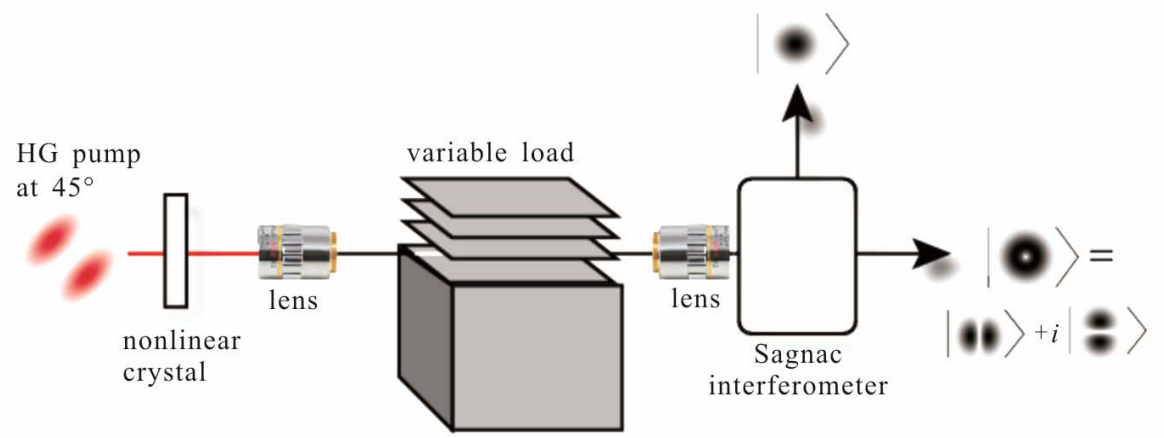

Figure 2. Proposed scheme to produce heralded single photons in arbitrary first-order transverse spatial states: $S$ represents a Sagnac interferometer. The stripped and stressed 3-mode fiber is pumped with a rotated HG pump laser. The HG laser pump additionally goes through a nonlinear crystal.

biphoton wavefunction from now on. In the basis of $\mathrm{HG}$ modes of the two photons it takes the form $([15,16])$

$$
\left|\Psi_{2 p h}\right\rangle=\left|\varphi_{n m}\right\rangle \otimes(|\mathrm{H}\rangle|\mathrm{V}\rangle+|\mathrm{V}\rangle|\mathrm{H}\rangle),
$$

where $|\mathrm{H}\rangle$ and $|\mathrm{V}\rangle$ denote the horizontal and vertical single-photon polarizations and $\left|\phi_{n m}\right\rangle$ denotes the spatial part of the biphoton wavefunction. This one we can further expand in terms of spatial $\mathrm{HG}$ modes but since we limit ourselves to the usage of 3-mode fibers we can immediately truncate the series and obtain [7]

$$
\left|\phi_{n m}\right\rangle=\left|\mathrm{HG}_{00}\right\rangle_{A}\left|\mathrm{HG}_{45^{\circ}}\right\rangle_{B} \otimes\left(|\mathrm{H}\rangle_{A}|\mathrm{~V}\rangle_{B}+|\mathrm{V}\rangle_{A}|\mathrm{H}\rangle_{B}\right) \text {. }
$$

$A$ and $B$ label the different angular momentum compnents of the wavefunction. Since the Sagnac interfereometer sorts different angular momentum components [7] these two labels also correspond to the different output ports of the interferometer.

Now we can use the results gained above. Using the compression stage in the setup depicted in Figure 2 we can induce stress and obtain the mode conversion from $\left|\mathrm{HG}_{45^{\circ}}\right\rangle$ to $\mathrm{LG}_{0}^{1}$ or $\mathrm{LG}_{0}^{-1}$ as shown in Equations (1) and (2).

Consequently the output photon in $A$ heralds an output photon in $B$ with a stress dependent well-defined firstorder spatial mode

$$
\left|\Psi_{B}\right\rangle=\left|\mathrm{LG}_{0}^{ \pm 1}\right\rangle \otimes(|\mathrm{H}\rangle|\mathrm{V}\rangle+|\mathrm{V}\rangle|\mathrm{H}\rangle) .
$$

Indeed these two different compressions possible allow to cover the entire Poincaré sphere of first-order transverse spatial modes [17].

\section{Conclusion}

In this paper we have generalized the results of [1] as we have worked with a polarized non-rotated beam and a true 3-mode fiber that has been stripped in the stress region. We have investigated different methods of fiber stripping and have shown that the method works even for a 3-mode fiber as needed for experimental proposals, e.g. in [7]. We investigated the heralding of single photons using our approach.

\section{Acknowledgements}

The author would like to thank C. C. Leary, M. G. Raymer and B. J. Smith for their hospitality at the University of Oregon and I. M. Deppner for many interesting discussions.

\section{REFERENCES}

[1] D. McGloin, N. B. Simpson and M. J. Padgett, "Transfer of Orbital Angular Momentum from a Stressed FiberOptic Waveguide to a Light Beam," Applied Optics, Vol. 37, No. 3, 1998, pp. 469-472. doi:10.1364/AO.37.000469

[2] L. Allen, M. W. Beijersbergen, R. J. C. Spreeuw and J. P. Woerdman, "Orbital Angular Momentum of Light and the Transformation of Laguerre-Gaussian Laser Modes," Physical Review A, Vol. 45, No. 11, 1992, pp. 8185-8189. doi:10.1103/PhysRevA.45.8185

[3] H. He, M. E. J. Friese, N. R. Heckenberg and H. Rubinsztein-Dunlop, "Direct Observation of Angular Momentum to Absorptive Particles from a Laser Beam with a Phase Singularity," Physical Review Letters, Vol. 75, No. 5, 1995, pp. 826-829. doi:10.1103/PhysRevLett.75.826

[4] M. E. J. Friese, J. Enger, H. Rubinsztein-Dunlop and N. R. Heckenberg, "Optical Angular Momentum Transfer to Trapped Absorbing Particles," Physical Review A, Vol. 54, No. 2, 1996, pp. 1593-1596. doi:10.1103/PhysRevA.54.1593

[5] N. B. Simpson, K. Dholakia, L. Allen and M. J. Padgett, "Mechanical Equivalence of Spin and Orbital Angular Momentum of Light: An Optical Spanner," Optics Letters, Vol. 22, No. 1, 1997, pp. 52-54. doi:10.1109/9.402235

[6] R. Čelechovský and Z. Bouchal, "Optical Implementation of the Vortex Information Channel," New Journal of Physics, Vol. 9, No. 9, 2007, p. 328. doi:10.1088/1367-2630/9/9/328

[7] C. C. Leary, L. A. Baumgardner and M. G. Raymer, "Stable Mode Sorting by Two-Dimensional Parity of 
Photonic Transverse Spatial States," Optics Express, Vol. 17, No. 4, 2009, pp. 2435-2452.

doi:10.1364/OE.17.002435

[8] M. Harris, C. A. Hill and J. M. Vaughan, "Optical Helices and Spiral Interference Fringes," Optics Communications, Vol. 106, No. 4-6, 1994, pp. 161-166. doi:10.1016/0030-4018(94)90314-X

[9] M. W. Beijersbergen, R. P. C. Coerwinkel, M. Kristensen and J. P. Woerdman, "Helical-Wavefront Laser Beams Produced with a Spiral Phaseplate," Optics Communications, Vol. 112, No. 5-6, 1994, pp. 321-327. doi:10.1016/0030-4018(94)90638-6

[10] G. A. Turnbull, D. A. Robertson, G. M. Smith, L. Allen and M. J. Padgett, "The Generation of Free-Space Laguerre-Gaussian Modes at Millimetre-Wave Frequencies by Use of a Spiral Phaseplate," Optics Communications, Vol. 127, No. 4-6, 1996, pp. 183-188. doi:10.1016/0030-4018(96)00070-3

[11] N. R. Heckenberg, R. McDuff, C. P. Smith and A. G. White, "Generation of Optical Phase Singularities by Computer-Generated Holograms," Optics Letters, Vol. 17, No. 3, 1992, pp. 221-223. doi:10.1364/OL.17.000221

[12] N. R. Heckenberg, R. McDuff, C. P. Smith, H. Rubinsztein-Dunlop and M. J. Wegener, "Laser Beams with Phase Singularities," Optical and Quantum Electronics,
Vol. 24, No. 9, 1992, pp. 951-962. doi:10.1007/BF01588597

[13] E. Abramochkin and V. Volostnikov, "Beam Transformations and Nontransformed Beams," Optics Communications, Vol. 83, No. 1-2, 1991, pp. 123-135. doi:10.1016/0030-4018(91)90534-K

[14] M. W. Beijersbergen, L. Allen, H. E. L. O. van der Veen and J. P. Woerdman, "Astigmatic Laser Mode Converters and Transfer of Orbital Angular Momentum," Optics Communications, Vol. 96, No. 1-3, 1993, pp. 123-132. doi:10.1016/0030-4018(93)90535-D

[15] P. G. Kwiat, K. Mattle, H. Weinfurter, A. Zeilinger, A. V. Sergienko and Y. Shih, "New High Intensity Source of Polarisation-Entangled Photon Pairs," Physical Review Letters, Vol. 75, No. 24, 1995, pp. 4337-4341. doi:10.1103/PhysRevLett.75.4337

[16] S. P. Walborn, S. Pádua and C. H. Monken, "Conservation and Entanglement of Hermite-Gaussian Modes in Parametric Down-Conversion," Physical Review A, Vol. 71, No. 5, 2005, p. 053812. doi:10.1103/PhysRevA.71.053812

[17] S. J. van Enk, "Geometric Phase, Transformations of Gaussian Light Beam and Angular Momentum Transfer," Optics Communications, Vol. 102, No. 1-2, 1993, pp. 5964. doi:10.1016/0030-4018(93)90472-H 